\title{
Dynamics Simulation on Vehicle Steering Mechanism
}

\author{
Lei Zhang ${ }^{1, a}$, En'guo Dong ${ }^{1, b}$
}

${ }^{1}$ Automotive and Transport School, Tianjin University of Technology and Education,

Tianjin, China, 300222

azldeg@126.com, bdegzl@sohu.com

Keywords: Steering Mechanism; Rigid-flexible Coupling model; Simulation;

\begin{abstract}
Using the multi-body system dynamic method, a steering mechanism simulation model is built and dynamics simulation analysis is studied. We have built a flexible tie-rod model and attained the natural modes using the finite element software of ANSYS. In this paper, a rigid-flexible coupling steering mechanism model is built using the software of ADAMS and ANSYS with the flexible tie-rod model. The torques of the trapezoidal steering arm during a bumping are analyzed. The dynamics characteristics of the multi-body rigid model and the rigid-flexible coupling model are compared. The accurate simulation results of the rigid-flexible coupling model were verified by simulation test.
\end{abstract}

\section{Introduction}

A conventional engineering simulation is based on a rigid body model, without considering an elastic deformation of mechanism. In fact, there is a larger elastic deformation on mechanism under big load or acceleration, deceleration condition, etc. as in [1-2]. Therefore the software of ADAMS and ANSYS can be combined to precisely simulate mechanism movement. A tie rod of trapezoidal steering mechanism is the component of easily appearing the elastic deformation; moreover the elastic deformation affects vehicle steering stability and tire wear. Therefore, in the trapezoidal steering mechanism design, the elastic deformation of a tie rod is considered and a flexible coupling of simulation model is built as in [3]. The dynamic performance of the steering mechanism in different condition is accurately analyzed.

\section{Tie rod model}

The steering mechanism is a full hydraulic steering system being composed of a whole trapezoidal steering arm, a steering power cylinder and a tie rod, etc. as in [4-5]. The tie rod consists of 3 parts as shown in Fig.1. Both ends of the tie rod are the tie rod joints, and the middle part is the main body of the tie rod.

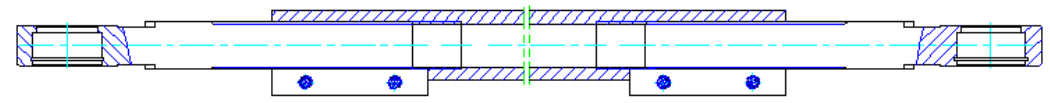

Fig.1 Tie rod

The main length of the tie rod is $1630 \mathrm{~mm}$, OD (the outer diameter) is $42 \mathrm{~mm}$, and ID (the inner diameter) is $39 \mathrm{~mm}$. The tie rod material is Q235, elastic modulus $E=2 \times 1011 \mathrm{~N} / \mathrm{m}^{2}$, and Poisson's ratio $u=0.3$, and density $\rho=7800 \mathrm{~kg} / \mathrm{m}^{3}$. The simplified finite element model is shown as in Fig.2.

The nature mode and vibration mode of the flexible tie rod is inherent from the dynamic characteristics parameters of it own structure. For a system with $n$ degrees of freedom, the free vibration equation is expressed

$$
M \ddot{X}+K X=0
$$

where, $M$ is the mass matrix, $K$ is the main stiffness matrix. The solution is as followings:

$$
X=A \sin (\omega t+\varphi)
$$

where, $A$ is as the amplitude array.

The acceleration array is derivative as equation (3)

$$
\ddot{X}=-\omega^{2} A \sin (\omega t+\varphi)
$$


Substitute equation (2) and equation (3) into equation (1), get equation (4) $\left(K-\omega^{2} M\right) A=0$

If equation (4) has non-zero solution, the condition is that coefficient determinant is equal to zero. Namely

$$
\operatorname{det}\left(K-\omega^{2} M\right)=0
$$

Equation (5) is the characteristic equation (or frequency equation). Expand it and get $n$-order algebraic equations about $\omega^{2}$, and the equation root is for the characteristic root. The corresponding eigenvector is called $i$-order natural mode. Each feature value and the corresponding eigenvectors are referred as the feature pairs.

To improve the computational efficiency of the dynamic simulation, the model order should not be too high, and there is reduction mode problem on the model. In reduction mode process, the main focus is to reduce the number of nature modes indicated elastic deformation. The nature modes of the 16-order on tie rod is selected, and the natural frequency is shown as in table 1.

Tab.1 Natural frequency

\begin{tabular}{|c|c|c|c|c|c|c|c|c|}
\hline Order & 1 & 2 & 3 & 4 & 5 & 6 & 7 & 8 \\
\hline Frequncy[HZ] & 2.06 & 2.51 & 2.51 & 2.73 & 4.35 & 4.74 & 230.9 & 230.9 \\
\hline Order & 9 & 10 & 11 & 12 & 13 & 14 & 15 & 16 \\
\hline Frequncy[HZ] & 622.77 & 622.77 & 1184.8 & 1184.8 & 1320.8 & 1887.9 & 1887.9 & 2129.3 \\
\hline
\end{tabular}

\section{Steering mechanism model}

A simulation model with the flexible tie-rod body is shown as in Fig.4. It is composed of the steering knuckle (two), the turning wheel (four), the suspension system (four), the steering trapezoid arm (two), the tie rod, the axles and other components. The suspension system is a candle suspension. It consists of the cylinder and the putting rod connected by a movement joint. The trapezoidal steering arm and the tie rod are connected with a ball joint. The flexible tie rod and the rigid tie rod joints are connected with a fixed joint.

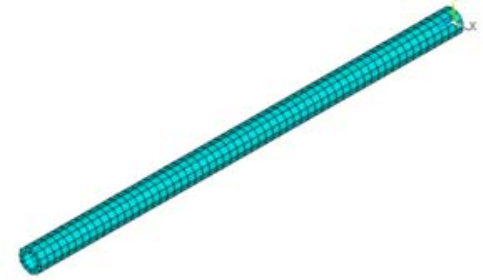

Fig.2 The finite element model of tie rod

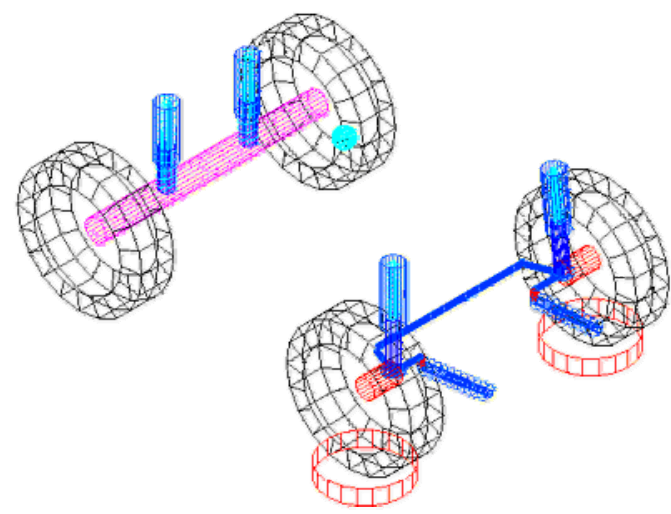

Fig.3 The flexible simulation model

\section{Simulation analyses}

\section{Dynamic characteristic analyses}

Figure 4 is the front-wheel-distance variation curves during a driving straight, while figure 5 is the swing angle curves during a bumping. As will be seen, the flexible body adds the wheel distance variation and the swing angle of the front wheel, and the stability of vehicle handling and the tire wear are destroyed. Therefore, to the steering mechanism design, the stiffness of the tie rod should be increased to improve vehicle stability and reduce the tire wear. 


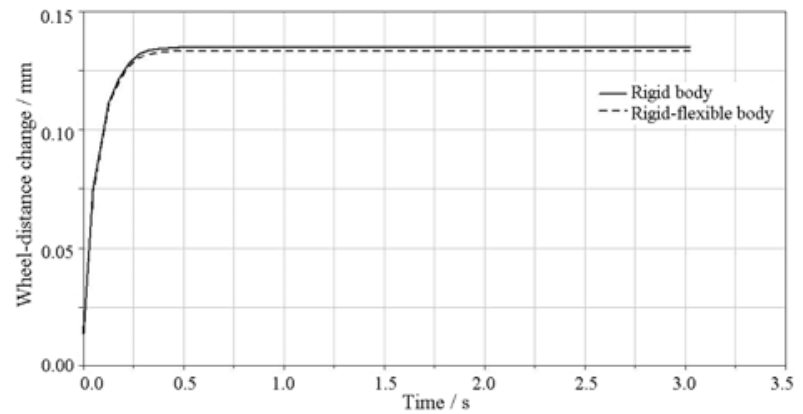

Fig.4 Wheel-distance variation

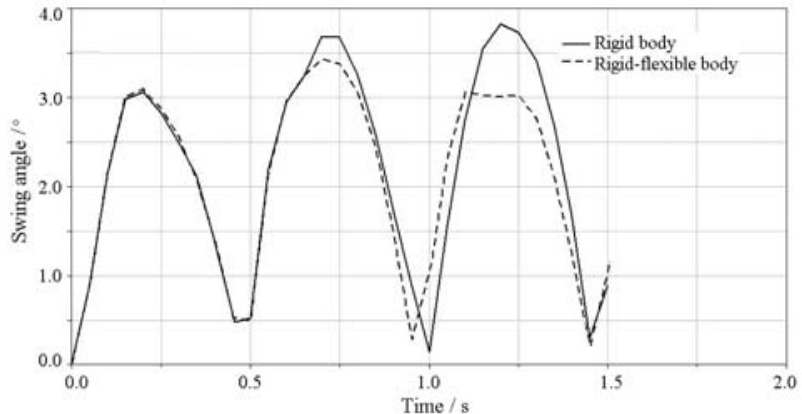

Fig.5 Swing angle variation

\section{Torque analysis}

In an early design process, the stress and strain of components are testified based on the steering torque. However in practical work condition, the vehicle is often driving on a bump road, and turning wheel is in a steering and bumping. Therefore the torque of steering mechanism is analyzed in the two conditions.

The torque withstood by the trapezoidal steering arm during a turning (from $0^{\circ}$ to the maximum turning angle) is shown as in Fig.6. In Fig.6, the solid line is shown on the multi-body model, and the dashed line is shown on the flexible coupling model. $X$-axis is forward direction, $Y$-axis is vertically upward direction, and $Z$-axis direction is the direction of the right side of driving. The force in the different simulation models is similar.

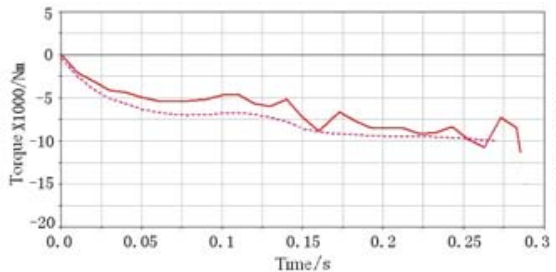

(a) $X$-axis direction

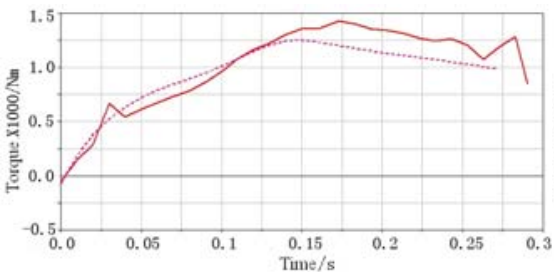

(b) $Y$-axis direction

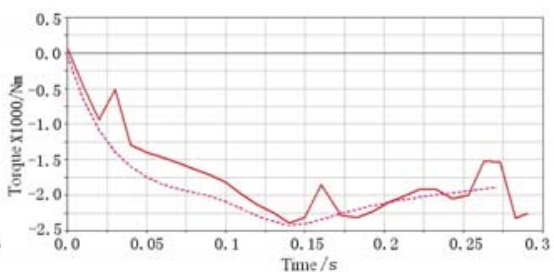

(c) Z-axis direction

Fig.6 Torques during a turning

The torque withstood by the trapezoidal steering arm during a bumping (the sine wave amplitude is $50 \mathrm{~mm}$ ) is shown as in Fig.7. Fig.7 (a-c) is the test curve of a rigid body model, and Fig.4 (d-f) is the test curve of a flexible coupling model.

For the rigid body model, the average torque of $X$-axis direction is $765 \mathrm{NM}$, with the $Y$-axis direction is $1972 \mathrm{NM}$, and the $Z$-axis direction is $1080 \mathrm{NM}$. For the flexible coupling model, the average torque of $X$-axis direction is $1184.4 \mathrm{NM}$, with the $Y$-axis is 51258NM, and the $Z$-axis direction is 28554NM. As will be seen, the torque of the flexible coupling model is far greater than the torque of the rigid model during a bumping. Therefore the design should be accord with the flexible model.

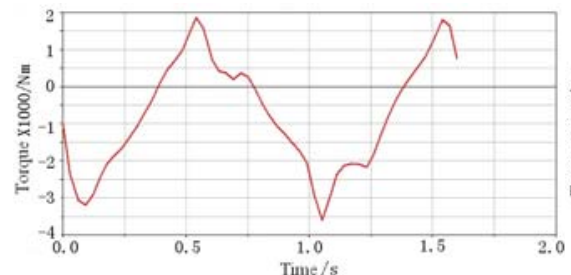

(a) $X$-axis direction

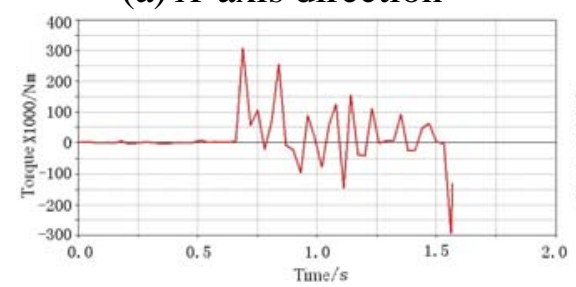

(d) $X$-axis direction

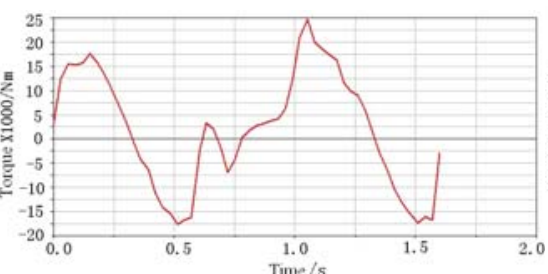

(b) $Y$-axis direction

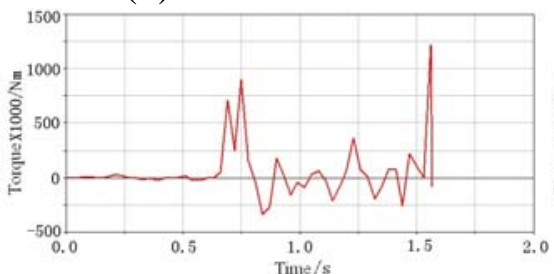

(e) $Y$-axis direction

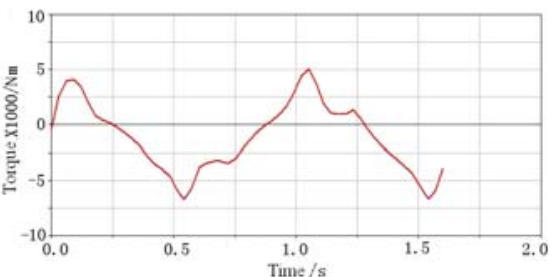

(c) Z-axis direction

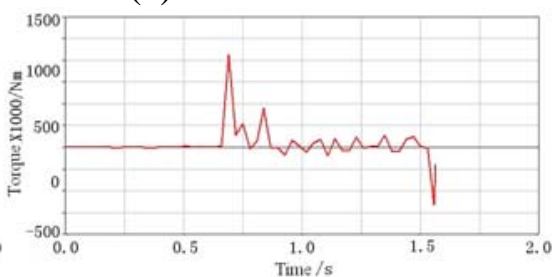

(f) $Z$-axis direction

Fig.7 Torques during a bumping 
The forces of the flexible tie rod in different conditions (the steering course, the driving straight course, and the bumping course) are shown as in table 2. As will be seen, the torque of the trapezoidal steering arm during a bumping is great. Therefore in the early design process, the stress of the steering mechanism should be calculated based on the bumping condition to avoid the earlier failure.

Tab.2 The force of tie rod

\begin{tabular}{|l|c|c|c|c|c|c|c|c|c|}
\hline \multirow{2}{*}{ Tie rod } & \multicolumn{3}{|c|}{ CASE $=1$} & \multicolumn{3}{c|}{ CASE $=2$} & \multicolumn{3}{c|}{ CASE = 3 } \\
\cline { 2 - 10 } & $\mathrm{F}_{\mathrm{X}}[\mathrm{N}]$ & $\mathrm{F}_{\mathrm{Y}}[\mathrm{N}]$ & $\mathrm{F}_{\mathrm{Z}}[\mathrm{N}]$ & $\mathrm{F}_{\mathrm{X}}[\mathrm{N}]$ & $\mathrm{F}_{\mathrm{Y}}[\mathrm{N}]$ & $\mathrm{F}_{\mathrm{Z}}[\mathrm{N}]$ & $\mathrm{F}_{\mathrm{X}}[\mathrm{N}]$ & $\mathrm{F}_{\mathrm{Y}}[\mathrm{N}]$ & $\mathrm{F}_{\mathrm{Z}}[\mathrm{N}]$ \\
\hline steering & -1041.7 & 112.3 & -68.8 & 453.8 & -120.5 & 171 & 200 & 285 & 69.9 \\
\hline driving & -0.075 & 13.3 & 69.6 & 437.9 & -2.649 & -0.4 & 13.2 & 13.2 & 70.7 \\
\hline bump & -2281780 & 823.4 & -62.5 & 4735 & 1220670 & 498703 & -596.7 & 45.2 & 268.4 \\
\hline \multirow{2}{*}{ Tie rod } & \multicolumn{3}{|c}{$\mathrm{CASE}=4$} & \multicolumn{3}{c|}{ CASE $=5$} & \multicolumn{3}{c|}{ CASE $=6$} \\
\cline { 2 - 11 } & $\mathrm{F}_{\mathrm{X}}[\mathrm{N}]$ & $\mathrm{F}_{\mathrm{Y}}[\mathrm{N}]$ & $\mathrm{F}_{\mathrm{Z}}[\mathrm{N}]$ & $\mathrm{F}_{\mathrm{X}}[\mathrm{N}]$ & $\mathrm{F}_{\mathrm{Y}}[\mathrm{N}]$ & $\mathrm{F}_{\mathrm{Z}}[\mathrm{N}]$ & $\mathrm{F}_{\mathrm{X}}[\mathrm{N}]$ & $\mathrm{F}_{\mathrm{Y}}[\mathrm{N}]$ & $\mathrm{F}_{\mathrm{Z}}[\mathrm{N}]$ \\
\hline steering & -181.5 & 71.5 & 405 & 143.4 & -138.2 & 93.6 & -1700 & 73.0 & -869.8 \\
\hline driving & 554 & 70.5 & 510.8 & 13.15 & 13.134 & 70.9 & 602.5 & 70.8 & 585.8 \\
\hline bump & 25360 & -269 & 34030 & 16.16 & -35.89 & 336 & 28599 & -38.6 & 25403 \\
\hline
\end{tabular}

\section{Conclusions}

A more realistic simulation model of the steering mechanism with a flexible tie rod is built using the software of ADAMS and ANSYS. The simulation analysis of the coupling steering mechanism can more truly reflect the vehicle dynamic characteristics. The stress based on the torque during a bumping is maximal in three vehicle conditions. The stiffness of the tie rod should be added to decrease the stress of the steering mechanism with the flexibility tie rod.

\section{Acknowledgements}

The research was funded by Chinese National Natural Science Foundation (51005167) and Tianjin University of Technology and Education Foundation (KJY11-07).

\section{Reference}

[1]SONG Shao-Yun, ZHANG Yong-Lin, YIN Fang. Design study on rigid-flexible coupled system based on virtual prototyping. Machinery, 2005, 32(1): 18-21

[2]HOU Hong-ling; ZHAO Yong-qiang, WEI Wei-feng. Kinetic Simulating and Analyzing Based on ADAMS and ANSYS. Modern Machinery, 2005(4): 62-63

[3]Xia Changgao, Gong Zhen. A Study on Vehicle Handling and Stability Based on Rigid-flexible Coupling Multi-body Model. Automotive Engineering, 2004, 26(5): 564-567

[4]DONG En-guo, ZHANG Lei. Optimization Design of Hydraulic Steering Mechanism of SGA3550 Truck. Tractor \& Farm Transporter, 2007, 34(1): 40-41, 44

[5]Dong Enguo, Zhang Lei, Shen Yanhua. Research on Front Wheel Alignment Parameters of Auto Based on Second Order Response Surface Model. Automobile technology, 2007, (11):32-36 